\title{
Analysis of orthotropic tensegrity plate strips using a continuum two-dimensional model
}

\author{
Paulina Obara ${ }^{1 *}$ \\ ${ }^{1}$ Politechnika Świętokrzyska, Wydział Budownictwa i Architektury, Al. Tysiąclecia PP 6, 25-314 Kielce, Poland
}

\begin{abstract}
A continuum two-dimensional model of orthotropic moderately thick plate strip is proposed in this paper. The model considers the different reference planes (lower, middle and upper) thus the different planes of support may be taken into account. The linear six-parameter and five-parameter shell theories are applied to the analysis. In case of a considered plate strip a number of parameters are reduced to four and to three respectively. The closed form of displacements and internal forces for any orthotropic plate strips are determined. As an example a continuum model of orthotropic tensegrity plate strip is considered. The influence of the self-stress state and some geometrical parameters on displacements are analyzed. The proposed approach can be used for all orthotropic systems.
\end{abstract}

\section{Introduction}

The concept of tensegrity structures covers trusses consisting of isolated compressed elements (struts) inside a continuous net of tensioned members (cables) $[1,2]$. The specificity of these structures lies in infinitesimal mechanisms balanced with self-stress states. The stiffness of these structures depends on the self-stress level. Tensegrity as a structural system offers many advantages over conventional structural systems.

The tensegrity concept has found applications within civil engineering, including bridges $[3,4]$, domes $[3,5]$ and shells [6]. The one of the widely investigated applications of tensegrity structures are double-layer tensegrity grids [7-10]. These systems consist with two parallel horizontal networks of members in tension forming the top and bottom layers. The grid nodes are linked by vertical and inclined bracing members in compression and tension. These systems can be treated as plate structures. Tensegrity plate structures can also be built with tensegrity modules, such as a simplex or an expanded octahedron [11-13].

The structural behaviour of tensegrity plate-like structures can be explained using a continuum threedimensional model [11-14]. This approach allows analyzing the influence of self-stress states on the displacements, strains and internal forces in the structures.

In the paper, the continuum model of orthotropic plate strips is considered. The two linear theories are applied to the analysis - six-parameter shell theory [15$21]$ and classical five-parameter shell theory. In analyzed systems there is no curvature, consequently a model is simpler than one shown in the mentioned papers. As a result, the two-dimensional model for moderately thick plate strips is obtained for both membrane and bending deformations. The model considers the different reference planes (lower, middle and upper) thus in the analysis the different planes of support may be taken into account. The proposed approach enables a direct use of engineering constants for the classical orthotropic continuum.

The model proposed in this paper can be used for all orthotropic systems, for example for orthotropic plates composed of tensegrity modules. A structural behaviour of tensegrity structures can be considered in a simple way. The influence of the self-stress state and some geometrical parameters on displacements can be analyzed.

\section{Material and methods}

An orthotropic moderately thick plate with width $a$ and constant thickness $h$ in the Cartesian coordinate system $\left(x_{1}, x_{2}, z\right)$ is considered. It is assumed that the plate is of an infinite extend in the $x_{2}$ direction (Fig. 1). The plate body is represented by reference surface $\Omega$. Three reference surfaces: the lower $\Omega^{L}$, the middle $\Omega^{M}$ and the upper $\Omega^{U}$ are taken into account:

$$
\begin{aligned}
& \Omega^{L}=\left\{x_{K}:\left(x_{1}, x_{2}\right) \in \Pi^{-}, z \in\langle 0 ; h\rangle\right\} \\
& \Omega^{M}=\left\{x_{K}:\left(x_{1}, x_{2}\right) \in \Pi, z \in\langle-h / 2 ; h / 2\rangle\right\} \\
& \Omega^{U}=\left\{x_{K}:\left(x_{1}, x_{2}\right) \in \Pi^{+}, z \in\langle-h ; 0\rangle\right\}
\end{aligned}
$$

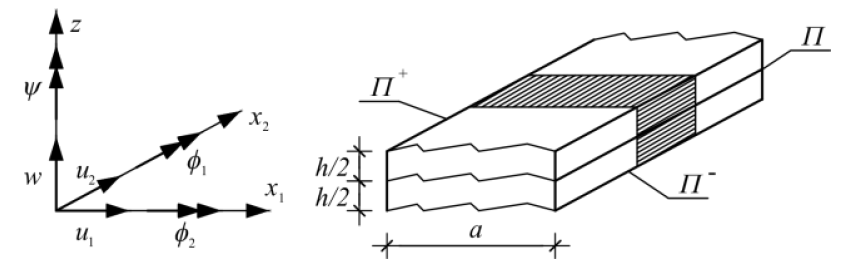

$\overline{{ }^{*} \text { Corresponding author: paula@tu.kielce.pl }}$ 
Fig. 1. Plate strip geometry and coordinate system.

It is assumed that translations and rotations are small, i.e., the linear theory can be used. In approach, the linear six-parameter and classical five-parameter shell theory is applied to the analysis. In plate there is no curvature, consequently the curvature of tensors $b_{\alpha \beta}$ and $b_{\alpha}^{\lambda}$, for $\alpha, \beta, \lambda=1,2$ are equal to zero.

In the first approach a kinematic model is formally equivalent to the Cosserat continuum [22-25] with six independent non-vanishing degrees of freedom: three translations $\left(u_{1}, u_{2}, w\right)$ and three rotations $\left(\phi_{1}, \phi_{2}, \psi\right)$. In the second $-\mathrm{a}$ drilling degree of freedom $\psi$ (rotation about the normal to the surface) is omitted. Both theories take into account the coupling of the membrane state and the bending state. In case of a considered plate strip a number of parameters are reduced to four and to three respectively.

\subsection{Linear four-parameter shell theory}

Implementation the six-parameter shell theory to the analysis of plate strip leads to four independent parameters describing the displacement field:

$$
\mathbf{q}\left(x_{1}\right)=\left\{\begin{array}{llll}
u_{1} & \psi & \phi_{1} & w
\end{array}\right\}^{T}
$$

The strain field, the internal forces and the external loads are as following:

$$
\begin{aligned}
& \boldsymbol{\varepsilon}\left(x_{1}\right)=\left\{\begin{array}{llll}
\gamma_{11} & \kappa_{13} & \kappa_{11} & \gamma_{13}
\end{array}\right\}^{T} \\
& \boldsymbol{\sigma}\left(x_{1}\right)=\left\{\begin{array}{llll}
N_{11} & M_{13} & M_{11} & N_{13}
\end{array}\right\}^{T} \\
& \mathbf{Q}\left(x_{1}\right)=\left\{\begin{array}{llll}
f_{1} & m_{3} & m_{1} & f_{3}
\end{array}\right\}^{T}
\end{aligned}
$$

The stress and strain vector reduced to two components $s_{1}, s_{5}$ and $e_{1}, e_{5}$. Consequently the constitutive equations, in Voight notation, for the linear theory of elasticity are expressed as:

$$
s_{1}=d_{11} e_{1}, \quad s_{5}=d_{55} e_{5}
$$

where

$$
e_{1}=\gamma_{11}+z \kappa_{11}, \quad e_{5}=\gamma_{13}+z \kappa_{13}
$$

Coefficients $d_{11}$ and $d_{55}$ are the components of the elastic matrix for orthotropic material. Below the next reduced relations and equations are showed:

- the kinematic relations:

$$
\gamma_{11}=\frac{d u_{1}}{d x_{1}}, \kappa_{13}=\frac{d \psi}{d x_{1}}, \kappa_{11}=\frac{d \phi_{1}}{d x_{1}}, \gamma_{13}=\phi_{1}+\frac{d w}{d x_{1}}
$$

- the internal forces:

$$
\begin{array}{ll}
N_{11}=\int_{z} s_{1} d z, & M_{13}=\int_{z} s_{5} z d z \\
M_{11}=\int_{z} s_{1} z d z, & N_{13}=\int_{z} s_{5} d z
\end{array}
$$

- the equilibrium equations:

$$
\begin{aligned}
& \frac{d N_{11}}{d x_{1}}+f_{1}=0, \quad \frac{d M_{13}}{d x_{1}}+m_{3}=0 \\
& \frac{d M_{11}}{d x_{1}}+N_{13}+m_{1}=0, \quad \frac{d N_{13}}{d x_{1}}+f_{3}=0
\end{aligned}
$$

Finally the equilibrium equations (8), in the nondimensional coordinate $\xi=x_{1} / a$, can be written as:

$$
\mathbf{L q}(\xi)=-a^{2} \mathbf{Q}(\xi)
$$

where:

$\mathbf{L}=\left[\begin{array}{cccc}A_{0} L_{1} & 0 & A_{1} L_{1} & 0 \\ 0 & B_{2} L_{1} & a B_{1} L_{2} & B_{2} L_{1} \\ A_{1} L_{1} & -a B_{1} L_{2} & -a^{2} B_{0}+A_{2} L_{1} & -a B_{0} L_{2} \\ 0 & B_{1} L_{1} & a B_{0} L_{2} & B_{0} L_{1}\end{array}\right]$

wherein $L_{1}=\frac{d^{2}}{d \xi^{2}}$ and $L_{2}=\frac{d}{d \xi}$ are the differential operators and parameters $A_{i}$ and $B_{i}$ depend on the reference surfaces (Table 1) and are expressed as:

$$
A_{i}=h_{i} d_{11}, \quad B_{i}=\alpha_{i} h_{i} d_{55} \quad \text { for } \quad i=0,1,2
$$

where $\alpha_{i}$ is the shear correction factor.

Table 1. Formulas of parameters depending on the reference surfaces.

\begin{tabular}{|c|c|c|c|}
\hline \multirow{2}{*}{ parameter } & \multicolumn{3}{|c|}{ surface } \\
\cline { 2 - 4 } & lower & middle & upper \\
\hline$h_{0}$ & \multicolumn{3}{|c|}{$h$} \\
\hline$h_{1}$ & $h^{2} / 2$ & 0 & $-h^{2} / 2$ \\
\hline$h_{2}$ & $h^{3} / 3$ & $h^{3} / 12$ & $h^{3} / 3$ \\
\hline$A_{0}$ & & $h d_{11}$ \\
\hline$A_{1}$ & $h^{2} d_{11} / 2$ & 0 & $-h^{2} d_{11} / 2$ \\
\hline$A_{2}$ & $h^{3} d_{11} / 3$ & $h^{3} d_{11} / 12$ & $h^{3} d_{11} / 3$ \\
\hline$B_{0}$ & \multicolumn{3}{|c|}{$\alpha_{0} h d_{55}$} \\
\hline$B_{1}$ & $\alpha_{1} h^{2} d_{55} / 2$ & 0 & $-\alpha_{1} h^{2} d_{55} / 2$ \\
\hline$B_{2}$ & $\alpha_{2} h^{3} d_{55} / 3$ & $\alpha_{2} h^{3} d_{55} / 12$ & $\alpha_{2} h^{3} d_{55} / 3$ \\
\hline
\end{tabular}

The first two equations in (9) describe the membrane state and the last two - the bending state. In general, these states are coupled. Solving the set of differential 
equations (9) it is possible to obtain the explicit functions of displacement:

$$
\begin{aligned}
& u_{1}(\xi)=C_{5}+C_{6} \xi+\frac{3 A_{1}}{a A_{0}} C_{4} \xi^{2}-\frac{a^{2}}{2 A_{0}} f_{1} \xi^{2}+\frac{a^{3} E_{1}}{6} f_{3} \xi^{3} \\
& \psi(\xi)=C_{7}+C_{8} \xi+\frac{a^{2}}{2}\left[D_{1} f_{3}-D_{0} m_{3}\right] \xi^{2} \\
& \phi_{1}(\xi)=-\frac{1}{a}\left[C_{2}+2 C_{3} \xi+3 C_{4} \xi^{2}+\frac{6}{a^{2} B_{0} E_{0}} C_{4}\right]+ \\
& +\frac{B_{1}}{a B_{0}} C_{8}-\frac{a}{B_{0}}\left(1+B_{1} D_{1}\right) f_{3} \xi-\frac{a^{3} E_{0}}{6} f_{3} \xi^{3}+a D_{1} m_{3} \xi+ \\
& +\frac{1}{B_{0}} m_{1}-\frac{A_{1}}{A_{0} B_{0}} f_{1} \\
& w(\xi)=C_{1}+C_{2} \xi+C_{3} \xi^{2}+C_{4} \xi^{3}+\frac{a^{4} E_{0}}{24} f_{3} \xi^{4}
\end{aligned}
$$

and internal forces:

$$
\begin{aligned}
& N_{11}(\xi)=-\frac{2 A_{1}}{a^{2}} C_{3}+\frac{A_{0}}{a} C_{6}-a f_{1} \xi+ \\
& -\frac{A_{1}}{B_{0}}\left(1+B_{1} D_{1}\right) f_{3}+A_{1} D_{1} m_{3} \\
& N_{13}(\xi)=-\frac{6}{a^{3} E_{0}} C_{4}-a f_{3} \xi-\frac{A_{1}}{A_{0}} f_{1}+m_{1} \\
& M_{11}(\xi)=-\frac{2 A_{2}}{a^{2}} C_{3}-\frac{6}{a^{2} E_{0}} C_{4} \xi+\frac{A_{1}}{a} C_{6}+ \\
& -\frac{a A_{1}}{A_{0}} f_{1} \xi+\frac{a^{2}}{2}\left(A_{1} E_{1}-A_{2} E_{0}\right) f_{3} \xi^{2}-\frac{A_{2}}{B_{0}}\left(1+B_{1} D_{1}\right) f_{3} \\
& +A_{2} D_{1} m_{3} \\
& M_{13}(\xi)=-\frac{6 B_{1}}{a^{3} B_{0} E_{0}} C_{4}+\frac{1}{a D_{0}} C_{8}+ \\
& +a\left(B_{1} D_{1}-B_{2} D_{2}\right) m_{3} \xi-\frac{A_{1} B_{1}}{A_{0} B_{0}} f_{1}+\frac{B_{1}}{B_{0}} m_{1}
\end{aligned}
$$

where:

$$
D_{i}=\frac{B_{i}}{B_{0} B_{2}-B_{1}^{2}}, E_{i}=\frac{A_{i}}{A_{0} A_{2}-A_{1}^{2}} \quad \text { for } \quad i=0,1,2
$$

The results for a plate strip can be used in analysis of different types of orthotropic systems, such as beams, plate strips, plates or of more complicated, multi-module plate structures. The displacement and internal force functions, of these systems, depend on components of the elastic matrix.

\subsection{Linear three-parameter shell theory}

In the classical five-parameter shell theory a drilling degree of freedom $\psi$ is not take into account. Consequently a drilling couple $M_{13}$ and an external load $m_{3}$ are omitted. Using this theory the behavior of the plate strip is described by three parameters being the subset of original ones:

$$
\widetilde{\mathbf{q}}\left(x_{1}\right)=\left\{\begin{array}{lll}
u_{1} & \phi_{1} & w
\end{array}\right\}^{T}
$$

and the strain field, the internal forces and the external loads (3) are given as follows:

$$
\begin{aligned}
& \widetilde{\boldsymbol{\varepsilon}}\left(x_{1}\right)=\left\{\begin{array}{lll}
\gamma_{11} & \kappa_{11} & \gamma_{13}
\end{array}\right\}^{T} \\
& \widetilde{\boldsymbol{\sigma}}\left(x_{1}\right)=\left\{\begin{array}{lll}
N_{11} & M_{11} & N_{13}
\end{array}\right\}^{T} \\
& \widetilde{\mathbf{Q}}\left(x_{1}\right)=\left\{\begin{array}{lll}
f_{1} & m_{1} & f_{3}
\end{array}\right\}^{T}
\end{aligned}
$$

The constitutive equations (4) are expressed as:

$$
s_{1}=\widetilde{d}_{11}\left(\gamma_{11}+z \kappa_{11}\right), \quad s_{5}=d_{55} \gamma_{13}
$$

where $\widetilde{d}_{11}$, in the case of the plane stress $\left(s_{3}=0\right)$, has the form:

$$
\widetilde{d}_{11}=d_{11}-\frac{d_{13}^{2}}{d_{33}}
$$

The equilibrium equations (8) can be written as:

$$
\widetilde{\mathbf{L}} \widetilde{\mathbf{q}}(\xi)=-a^{2} \widetilde{\mathbf{Q}}(\xi)
$$

where:

$$
\widetilde{\mathbf{L}}=\left[\begin{array}{ccc}
A_{0} L_{1} & A_{1} L_{1} & 0 \\
A_{1} L_{1} & A_{2} L_{1}-a^{2} B_{0} & -a B_{0} L_{2} \\
0 & a B_{0} L_{2} & A_{1} L_{1}
\end{array}\right]
$$

Solving the set of differential equations (18) it is possible to obtain the explicit functions of displacements:

$$
\begin{aligned}
u_{1}(\xi)= & C_{5}+C_{6} \xi+\frac{3 A_{1}}{a A_{0}} C_{4} \xi^{2}-\frac{a^{2}}{2 A_{0}} f_{1} \xi^{2}+\frac{a^{3} E_{1}}{6} f_{3} \xi^{3} \\
\phi_{1}(\xi)= & -\frac{1}{a}\left[C_{2}+2 C_{3} \xi+3 C_{4} \xi^{2}+\frac{6}{a^{2} B_{0} E_{0}} C_{4}\right]+ \\
& -\frac{a}{B_{0}} f_{3} \xi-\frac{a^{3} E_{0}}{6} f_{3} \xi^{3}+\frac{1}{B_{0}} m_{1}-\frac{A_{1}}{A_{0} B_{0}} f_{1} \\
w(\xi)= & C_{1}+C_{2} \xi+C_{3} \xi^{2}+C_{4} \xi^{3}+\frac{a^{4} E_{0}}{24} f_{3} \xi^{4}
\end{aligned}
$$

and internal forces:

$$
\begin{aligned}
N_{11}(\xi) & =-\frac{2 A_{1}}{a^{2}} C_{3}+\frac{A_{0}}{a} C_{6}-a f_{1} \xi-\frac{A_{1}}{B_{0}} f_{3} \\
N_{13}(\xi) & =-\frac{6}{a^{3} E_{0}} C_{4}-a f_{3} \xi-\frac{A_{1}}{A_{0}} f_{1}+m_{1} \\
M_{11}(\xi) & =-\frac{2 A_{2}}{a^{2}} C_{3}-\frac{6}{a^{2} E_{0}} C_{4} \xi+\frac{A_{1}}{a} C_{6}-\frac{a A_{1}}{A_{0}} f_{1} \xi+ \\
& -\frac{A_{2}}{B_{0}} f_{3}-\frac{a^{2}}{2} f_{3} \xi^{2}
\end{aligned}
$$

\subsection{Orthotropic tensegrity plate strip}

To illustrate the proposed approach, a continuum orthotropic model of the tensegrity plate-like structure 
[11-13], which could be supported both on internal or external surfaces, is used. A system of repeating expanded four-strut octahedron modules is used as an example (Fig. 2). The system is orthotropic for the following geometric parameters $x / X=0.65, y / Y=0.3$ and $z / Z=0.56$. For tensegrity systems, the components of the elastic matrix depend on cables $\left(E A_{\text {cable }}\right)$ and struts stiffness $\left(E A_{\text {struts }}\right)$ and on the level of self-stress $\sigma$.

$$
n=\frac{(E A)_{\text {cable }}}{(E A)_{\text {struct }}}, \quad \sigma=\frac{S}{(E A)_{\text {struct }}}
$$

where $E$ is the Young's modulus, $A$ is the cross sections and $S$ is the axial force. The coefficient $n$ describes the proportions of member properties and $\sigma$ describes the level of self-stress in tensegrity structures. The self-stress state is the most important feature of tensegrity structures. This state makes structure as strong as the self-supporting structure and stabilizes the infinitesimal mechanisms occurred in tensegrity structures.

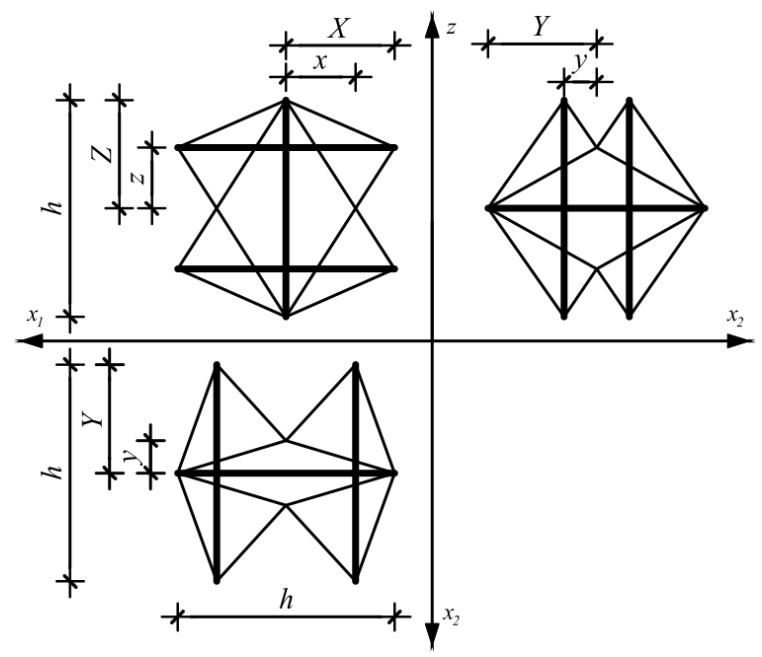

Fig. 2. Expanded four-strut octahedron.

The appropriate coefficients of the elastic matrix for plate strip consisting of octahedron modules are as follows [13]:

$d_{11}=\frac{2 E A}{h^{2}} \delta_{11}, \quad d_{33}=\frac{2 E A}{h^{2}} \delta_{33}, \quad d_{55}=d_{13}=\frac{E A}{h^{2}} \delta_{13}$

where:

$$
\begin{aligned}
& E A=(E A)_{\text {struct }} \\
& \delta_{11}=1+1.52325 n+0.129225 \sigma \\
& \delta_{13}=1.26604 n-0.153207 \sigma \\
& \delta_{33}=1+0.921194 n+0.16101 \sigma
\end{aligned}
$$

\section{Result and discussion}

The plate strips with different kinds of boundary conditions and different planes of support are discussed. The results of the analysis are described using $L$ for the lower surface, $M$ for the middle surface and $U$ for the upper surface. The closed form of the displacements and the internal forces for any orthotropic plate strip are determined.

First, the plate strips loaded by concentrated forces (axial and vertical), concentrated moments and mass forces are considered. Secondly, the plate strip under sinusoidal load is studied.

Based on the developed formulas the maximal displacements for tensegrity plate strips are calculated. The influence of the self-stress state, some geometrical parameters and planes of support is analyzed.

The proposed approach enables a direct use of engineering constants for the classical orthotropic continuum.

\subsection{Closed form of displacements and internal forces}

The closed form of the displacements and the internal forces for plate strips are determined. Two methods are applied. The first approach based on equations (12) and (13) second approach - on (20) and (21). These formulas depend on boundary conditions. Three kinds of support, i.e., cantilever (Fig. 3a), clamped-clamped (Fig. 3b) and simply supported (Figs. 3c, 3d) are considered.

For the cantilever plate strip three cases of load are taken into account: case $c 1-$ load by axial force $F_{1}$, case $c 2$ - load by vertical force $F_{2}$ and case $c 3$ - load by mass force $f_{3}(\xi)=-q_{0}$. For the clamped-clamped plate strip only load by force mass $f_{3}(\xi)=-q_{0}$ (case $c c 1$ ) is studied. For the simply supported plate strip three cases of load are taken into account: case $s 1$ - load by bending moment $M$, case $s 2-$ load by mass force $f_{3}(\xi)=-q_{0}$ and case $s 3$ - sinusoidal load $f_{3}(\xi)=-q_{0} \sin (\pi \xi)$. For the last one case, the equations (9) and (18) are solving by applying trigonometric functions that satisfying the boundary conditions for simply supported plate.

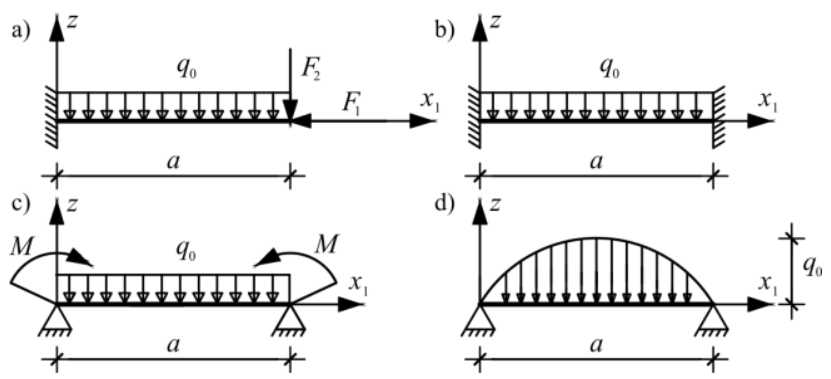

Fig. 3. Analysed plate strips: a) cantilever, b) clampedclamped, c), d) simply supported.

\subsubsection{Linear four-parameter shell theory}

Using the four-parameter shell theory the formulas of internal forces are received. For all plate strips the internal forces do not depend on geometric and physical properties of orthogonal structures. These forces almost always do not depend on the reference surface and obtained formulas are known from literature. Only for simply supported plate strip, independently from case of load, the constant axial force, which depend on the 
reference surface and on the plate thickness $h$, occurs additionally. For example, for case $s 1$ is received:

$$
N_{11}^{L}(\xi)=-N_{11}^{U}(\xi)=-\frac{3 M}{2 h}, \quad N_{11}^{M}(\xi)=0
$$

and for case $s 2$ :

$$
N_{11}^{L}(\xi)=-N_{11}^{U}(\xi)=-\frac{a^{2} q_{0}}{8 h}, \quad N_{11}^{M}(\xi)=0
$$

The formulas of all displacement $\left(u_{1}, \psi, \phi_{1}, w\right)$ for all plate strips depend on properties of orthogonal structures and on the reference surface. Decoupling of the bending and the membrane behaviour occurs only for the middle surface. For example, the closed form of the maximum displacements are as follows:

- case $c 1$ (Fig. $3 \mathrm{a}-F_{2}=0, q_{0}=0$ ):

$u_{\max }^{M}=-\frac{a F_{1}}{h} \frac{1}{d_{11}}, \quad u_{\max }^{L}=u_{\max }^{U}=-\frac{4 a F_{1}}{h} \frac{1}{d_{11}}$

- case $c 2$ (Fig. $3 \mathrm{a}-F_{1}=0, q_{0}=0$ ):

$$
\begin{aligned}
& w_{\max }^{M}=-\frac{a^{3} F_{2}}{h^{3}}\left[4 \frac{1}{d_{11}}+\frac{1}{\alpha_{0}}\left(\frac{h}{a}\right)^{2} \frac{1}{d_{55}}\right] \\
& w_{\max }^{L}=w_{\max }^{U}=-\frac{a^{3} F_{2}}{h^{3}}\left[4 \frac{1}{d_{11}}+4 \frac{\alpha_{2}}{\alpha_{3}}\left(\frac{h}{a}\right)^{2} \frac{1}{d_{55}}\right]
\end{aligned}
$$

- case $c 3$ (Fig. $3 \mathrm{a}-F_{1}=0, F_{2}=0$ ):

$$
\begin{aligned}
& w_{\max }^{M}=-\frac{a^{4} q_{0}}{8 h^{3}}\left[12 \frac{1}{d_{11}}+4 \frac{1}{\alpha_{0}}\left(\frac{h}{a}\right)^{2} \frac{1}{d_{55}}\right] \\
& w_{\text {max }}^{L}=w_{\max }^{U}=-\frac{a^{4} q_{0}}{8 h^{3}}\left[12 \frac{1}{d_{11}}+16 \frac{\alpha_{2}}{\alpha_{3}}\left(\frac{h}{a}\right)^{2} \frac{1}{d_{55}}\right]
\end{aligned}
$$

- case $c c 1$ (Fig. 3b):

$$
\begin{aligned}
& w_{\text {max }}^{M}=-\frac{a^{4} q_{0}}{8 h^{3}}\left[\frac{1}{4} \frac{1}{d_{11}}+\frac{1}{\alpha_{0}}\left(\frac{h}{a}\right)^{2} \frac{1}{d_{55}}\right] \\
& w_{\text {max }}^{L}=w_{\text {max }}^{U}=-\frac{a^{4} q_{0}}{8 h^{3}}\left[\frac{1}{4} \frac{1}{d_{11}}+4 \frac{\alpha_{2}}{\alpha_{3}}\left(\frac{h}{a}\right)^{2} \frac{1}{d_{55}}\right]
\end{aligned}
$$

- case $s 1$ (Fig. $3 \mathrm{c}-q_{0}=0$ ):

$w_{\max }^{M}=-\frac{3 a^{2} M}{2 h^{3}} \frac{1}{d_{11}}, w_{\text {max }}^{L}=w_{\text {max }}^{U}=-\frac{3 a^{2} M}{8 h^{3}} \frac{1}{d_{11}}$

- case $s 2$ (Fig. $3 \mathrm{c}-M=0$ ):

$$
\begin{aligned}
& w_{\text {max }}^{M}=-\frac{a^{4} q_{0}}{8 h^{3}}\left[\frac{5}{4} \frac{1}{d_{11}}+\frac{1}{\alpha_{0}}\left(\frac{h}{a}\right)^{2} \frac{1}{d_{55}}\right] \\
& w_{\text {max }}^{L}=w_{\text {max }}^{U}=-\frac{a^{4} q_{0}}{8 h^{3}}\left[\frac{1}{2} \frac{1}{d_{11}}+4 \frac{\alpha_{2}}{\alpha_{3}}\left(\frac{h}{a}\right)^{2} \frac{1}{d_{55}}\right]
\end{aligned}
$$

- case $s 3$ (Fig. 3d):

$$
\begin{aligned}
& w_{\max }^{M}=-\frac{a^{4} q_{0}}{\pi^{4} h^{3}}\left[12 \frac{1}{d_{11}}+\pi^{2} \frac{1}{\alpha_{0}}\left(\frac{h}{a}\right)^{2} \frac{1}{d_{55}}\right] \\
& w_{\max }^{L}=-\frac{a^{4} q_{0}}{\pi^{4} h^{3}}\left[12 \frac{1}{d_{11}}+4 \pi^{2} \frac{\alpha_{2}}{\alpha_{3}}\left(\frac{h}{a}\right)^{2} \frac{1}{d_{55}}\right] \\
& w_{\max }^{U}=w_{\text {max }}^{L}
\end{aligned}
$$

For plates in which the transverse force is not occurred (cases $c 1$ and $s 1$ ) the displacements do not depend on the shear factors $\alpha_{i}$. For these plates the displacement depend only on one component of the elastic matrix $d_{11}$.

For others plates the displacements depend on three shear correction factors $\alpha_{i}(\mathrm{i}=0,1,2)$ and on the parameter:

$$
\alpha_{3}=4 \alpha_{0} \alpha_{2}-3 \alpha_{1}^{2}
$$

However if the middle references surface is considered only one shear factor $\alpha_{0}$ occurs. The influence shear factors depends on the component of the elastic matrix $d_{55}$ and on proportion thickness to length $(h / a)$ - the thicker plate the bigger influence.

The values of the shear correction factors are established for the respective transverse shear stress resultants and stress couples [26-28]. The determination of these factors is not the aim of this paper. To illustrate influence of the reference plane on behaviour of tensegrity structures it is assumed the following values of the shear factors: $\alpha_{0}=5 / 6, \alpha_{2}=7 / 10$ [26-28] and $\alpha_{1}=8 / 10$. Value of the last correction factor is assumed so that the parameter (34) is positive definite.

\subsubsection{Linear three-parameter shell theory}

Using the three-parameter shell theory, for plates in which the transverse force is occurred, the displacements depend on the shear factors $\alpha_{0}$. The factors $\alpha_{i}(\mathrm{i}=1,2,3)$ is not occurred in this approach. In all cases the horizontal displacement $u_{1}$ depends on the reference surface. For all cantilever and clamped-clamped plate strips and for the simply supported plate strip under sinusoidal load the rotation $\phi_{1}$ and the deflection $w$ do not depend on the reference surface. The formulas of maximum displacement for these cases are equal:

$$
w_{\max }^{L}=w_{\max }^{U}=w_{\max }^{M}
$$

and there are the same like in equations: $(28)_{1},(29)_{1}$, $(30)_{1}$ and (33) $)_{1}$ respectively. Only the parameter $d_{11}$ is equal $\widetilde{d}_{11}$ according to (17). In two cases of the simply supported plate strip ( $s 1$ and $s 2)$ the displacements depend on the reference surface:

- case $s 1$ (Fig. $3 \mathrm{c}-q_{0}=0$ ): 
$w_{\max }^{M}=-\frac{3 a^{2} M}{2 h^{3}} \frac{1}{\widetilde{d}_{11}}, w_{\text {max }}^{L}=w_{\text {max }}^{U}=-\frac{3 a^{2} M}{8 h^{3}} \frac{1}{\widetilde{d}_{11}}$

- case $s 2$ (Fig. $3 \mathrm{c}-M=0)$ :

$$
\begin{aligned}
& w_{\max }^{M}=-\frac{a^{4} q_{0}}{8 h^{3}}\left[\frac{5}{4} \frac{1}{\widetilde{d}_{11}}+\frac{1}{\alpha_{0}}\left(\frac{h}{a}\right)^{2} \frac{1}{d_{55}}\right] \\
& w_{\max }^{L}=w_{\max }^{U}=-\frac{a^{4} q_{0}}{8 h^{3}}\left[\frac{1}{2} \frac{1}{\widetilde{d}_{11}}+\frac{1}{\alpha_{0}}\left(\frac{h}{a}\right)^{2} \frac{1}{d_{55}}\right]
\end{aligned}
$$

\subsection{Analysis of orthotropic tensegrity plate strip}

Based on the developed formulas the maximal displacements for tensegrity plate strips are calculated. The displacements as a function of parameters (24) and, in consequence, the coefficients (22), are determined The influence of the self-stress level $\sigma$ and the stiffness of cables and struts $n$ on displacements in tensegrity plate strip can be estimated in a simple way.

The results of parametric analysis are shown in Figures 4-8. The dependence of maximum displacement on the self-stress level $\sigma$ is presented. Two cases of thickness to length ratio for moderately thick plate strips, $h / a=0.1 \quad$ (Fig. 4a-8a) and $h / a=0.25$ (Fig. 4b-8b), are considered. Two cases of parameter $n(22)_{1}$ are taking into account: $n=0.2$ (continuous line -) and $n=0.7$ (dotted line ---). Results for middle surface are determined by $(\bullet)$ and for upper (or lower) surface by $(\boldsymbol{\Delta})$. Depending on the analyzed case the results are presented in normalized form:

$$
C_{\mathrm{W}}=-\frac{a^{3} F_{2}}{E A h} \quad \text { or } \quad C_{\mathrm{R}}=-\frac{a^{4} q_{0}}{8 E A h}
$$

a)

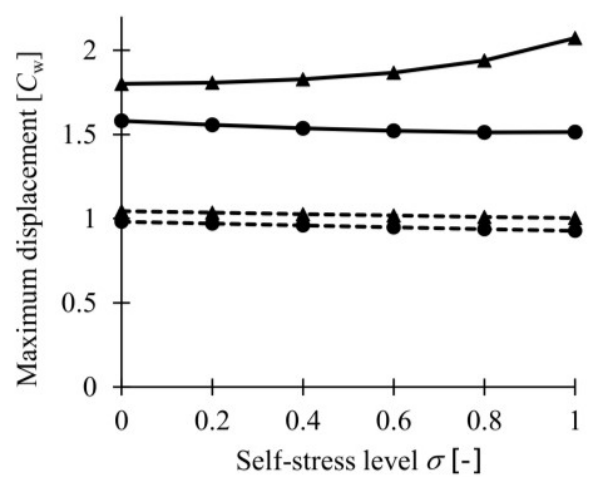

b)

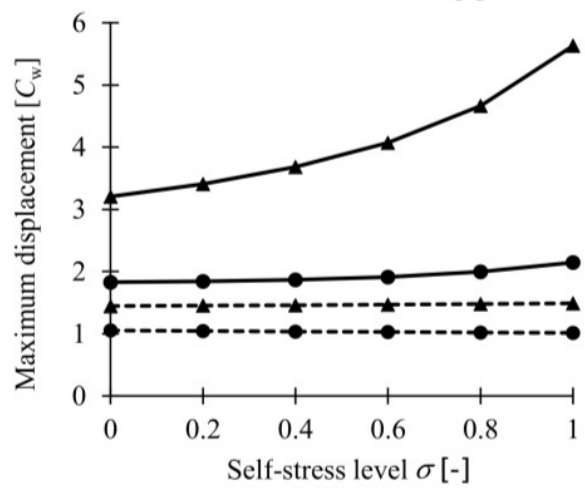

Fig. 4. Maximum displacement for case $c 2$ : a) $h / a=0.1$, b) $h / a=0.25$.

a)

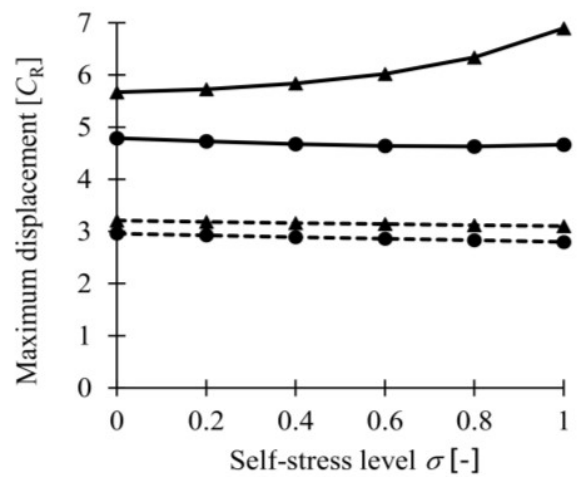

b)

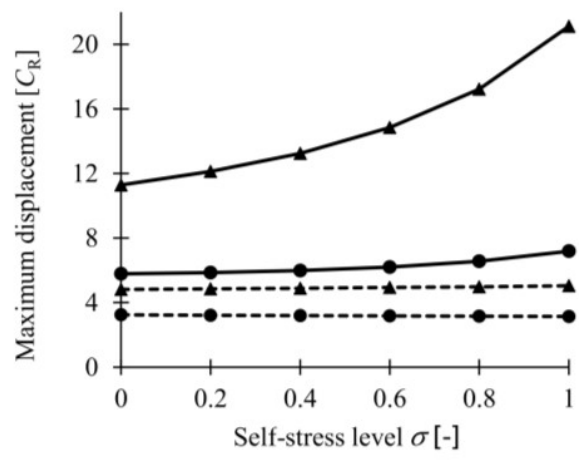

Fig. 5. Maximum displacement for case $c 3$ : a) $h / a=0.1$, b) $h / a=0.25$.

a)

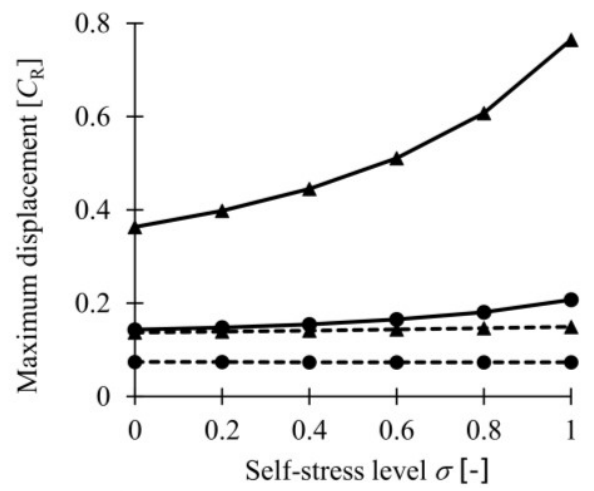

b)

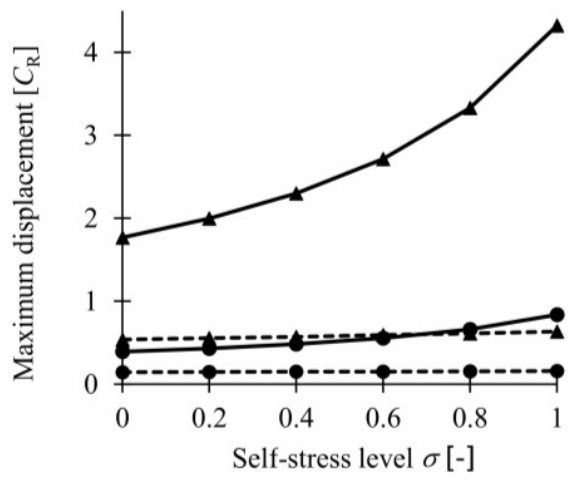

Fig. 6. Maximum displacement for case $c c 1$ : a) $h / a=0.1$, b) $h / a=0.25$.

Comparing obtained results of the analysis it can be noticed: 
- the influence of self-stress level on displacements decreases with increasing the stiffness of cables (the parameter $n$ increases),

- the influence of self-stress level is bigger when the upper (or lower) surface is considered,

- the thickness to length ratio significantly influence on displacements.

a)

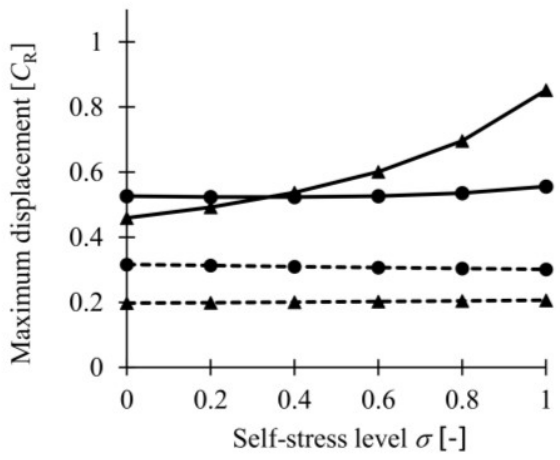

b)

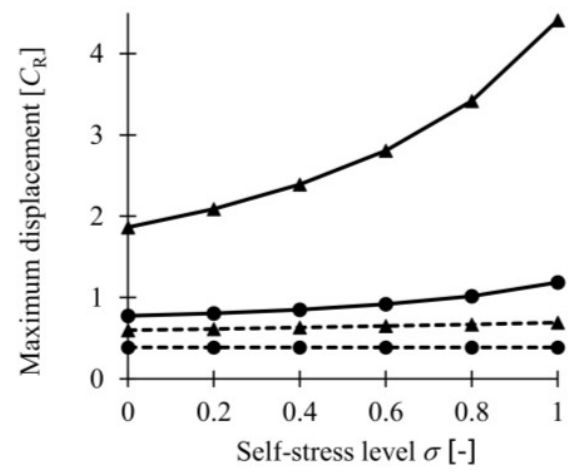

Fig. 7. Maximum displacement for case $s 2$ : a) $h / a=0.1$, b) $h / a=0.25$.

a)

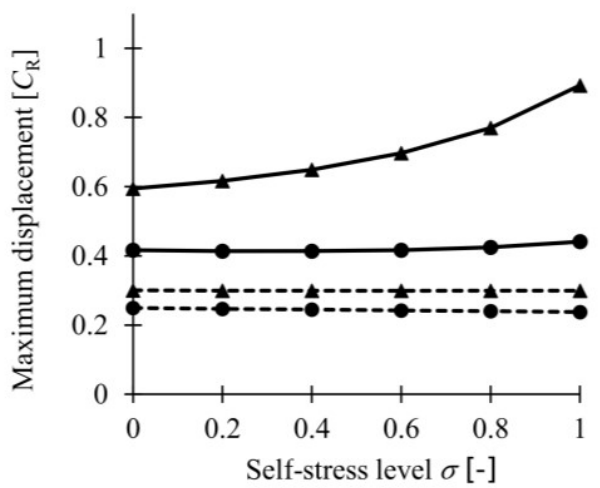

b)

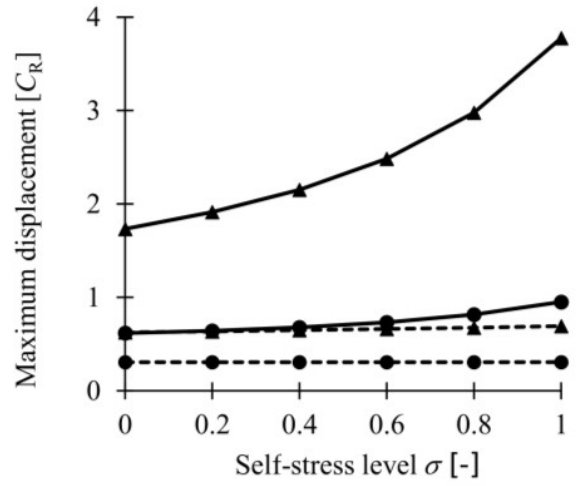

Fig. 8. Maximum displacement for case $s 3$ : a) $h / a=0.1$, b) $h / a=0.25$.

\section{Conclusion}

In the paper, the continuum model of orthotropic plate strips are considered. The linear four-parameter shell theory and classical three-parameter shell theory are proposed to determine the displacements and the internal forces. The obtaining closed form can be useful in the design process and construction of different types of orthotropic moderately thick plate strips. The different planes of support (lower, middle and upper) may be taken into account. The proposed approach enables a direct use of engineering constants for the classical orthotropic continuum.

The proposed model can be used for analysis of the orthotropic plate strip composed of tensegrity modules. Using the continuum model helps understand the unique properties and structural behaviour of tensegrity structures. In the analysis, the self-stress and selected geometrical parameters of tensegrity are introduced. Additionally, this approach simplifies calculations, that is, it is not necessary to describe the whole complex tensegrity structures with the use of computational methods.

The proposed approach can be used for all orthotropic systems having practical applications in civil engineering.

\section{References}

1. R. Motro, Tensegrity: structural systems for the future (Kogan Page, London, 2003)

2. R.E. Skelton, M.C. de Oliveira, Tensegrity systems (Springer, London, 2009)

3. V. Gómez-Jáuregui, Tensegrity structures and their application to architecture (Servicio de Publicaciones de la Universidad de Cantabria, 2010)

4. A. Kasprzak, A possibility of using tensegrity in bridge structures (Ph.D. Thesis, Warsaw University of Technology, 2014)

5. D. Campbell, D. Chen, P. Gossen, K. Hamilton, Effects of Spatial Triangulation on the Behavior of "Tensegrity" Domes, Spatial, Lattice and Tension Structures (ASCE, New York, 1994)

6. S.M.L. Adriaenssens, M.R. Barnes, Eng Struct, 23, 29-36 (2001)

7. V. Gomez-Jauregui, R. Arias, C. Otero, C. Manchado, Int J Space Struct 27, 155-166 (2012)

8. Y. Kono, K. K. Choong, T. Shimada, H. Kunieda, J IASS 40, 130, 103-111 (1999)

9. W. Gilewski, J. Kłosowska, P. Obara, Procedia Engineering 153, 173-179 (2016)

10. L. Crawfordt, Transgender architectonics: the shape of change in modernist space (Routledge, 2016) 
11. A. Al Sabouni-Zawadzka, W. Gilewski, Advances in Mechanics: Theoretical, Computational and Interdisciplinary Issues, (CRC Belkema, Taylor \& Francis Group, London, 197-200, 2016)

12. A. Al Sabouni-Zawadzka and W. Gilewski, Procedia Engineering 153, 887-894 (2016).

13. A. Al Sabouni-Zawadzka, W. Gilewski, J. Kłosowska, P. Obara, Eng. Trans. 64, 4, 501-508 (2016)

14. K. Kebiche, M.N. Kazi Aoual, R. Motro, , Int J Space Struct 23, 103-115 (2008)

15. J. Chróścielewski, J. Makowski, W. Pietraszkiewicz, Statics and dynamics of multifold shell: nonlinear theory and finite element method (IPPT PAN, Warsaw, 2004)

16. W. Pietraszkiewicz, ZAMM Z. Angew. Math. Mech. 96, 8, 899-915 (2016)

17. W. Witkowski, Synthesis of formulation of nonlinear mechanics of shells undergoing finite rotations in the context of FEM (Politechnika Gdańska, Gdańsk, 2011).

18. S. Burzyński, J. Chróścielewski, K. Daszkiewicz, W. Witkowski, "Geometrically nonlinear FEM analysis of FGM shells based on neutral physical syrface approach in 6-paramiter shell theory", Compos. Part B 107, 203-213 (2016)

19. J. Chróścielewski, W. Pietraszkiewicz, W. Witkowski, ZAMM Z. Angew. Math. Mech. 96, 2, 191-204 (2016)

20. J. Chróścielewski, A. Sabik, B. Sobczyk, W. Witkowski, Thin-Walled Struct. 105, 2, 207-219 (2016)

21. J. Chróścielewski, I. Kreja, A. Sabik, W. Witkowski, Mech. Adv. Mater. Struct. 18, 403419 (2011)

22. W. Pietraszkiewicz, V. Eremeyev, Int. J. Solids Struct. 46, 774-787 (2009)

23. S. Burzyński, Inż. Morska Geotech. 2, 125-134 (2013)

24. W. Pietraszkiewicz, V. Konopińska, Int. J. Solids Struct. 51, 2133-2143 (2014)

25. W. Nowacki, Irreversible Aspects of Continuum Mechanics and Transfer of Physical Characteristics in Moving Fluids (IUTAM Symposia Vienna, 259278, 1966)

26. J. Chróścielewski, J. Makowski, H. Stumpf, Comput. Meth. Appl. Mech. Eng. 41, 1-46 (1997)

27. W. Pietraszkiewicz, (Polish Scientific Publishers, Warsaw, Poznań 1979)

28. J. Chróścielewski, W. Pietraszkiewicz, W. Witkowski, Int. J. Solids Struct. 47, 3537-3545 (2010) 